\title{
Plasma lipids can be reliably assessed within 24 hours after acute myocardial infarction
}

\author{
M. Sewdarsen, S. Vythilingum, I. Jialal* and R. Nadar \\ Ischaemic Heart Disease Clinic, R. K. Khan Hospital and Department of Chemical Pathology, University of \\ Natal Medical School, Durban, South Africa.
}

\begin{abstract}
Summary: Total serum cholesterol and triglycerides were measured in 159 Indian patients (134 males) with acute myocardial infarction during their stay in hospital (days 1 and 2) and 3 months later in order to assess whether lipid levels measured soon after acute myocardial infarction represent basal values. Early and 3 month lipid levels were also compared according to the sex and glucose tolerance of the patients.

The mean total cholesterol levels on admission (day 1) were comparable to the 3 month values in both men $(6.09 \pm 0.10$ vs $6.18 \pm 0.09 \mathrm{mmol} / \mathrm{l})$ and women $(6.75 \pm 0.30$ vs $6.44 \pm 0.22 \mathrm{mmol} / \mathrm{l})$ irrespective of glucose tolerance. In the entire group cholesterol levels on day 2 were significantly lower than the admission and 3 month values. Nevertheless there was a significant correlation between day 2 and 3 month values $(P<0.0005)$.

Serum triglyceride levels on day 2 were influenced by glucose tolerance and were significantly lower than the 3 month values in patients with normal glucose tolerance but not in patients with abnormal glucose tolerance. However there was a significant correlation between day 2 triglyceride values in patients with both normal and abnormal glucose tolerance.

These results suggest that serum cholesterol measured on admission after acute myocardial infarction may be reliably used to represent basal values in both men and women irrespective of glucose tolerance. Although cholesterol and triglycerides measured on day 2 vary with either the gender or glucose tolerance of the patients, these values are still a useful guide to the patients' basal lipid state.
\end{abstract}

Introduction

The presence of hyperlipidaemia is usually not assessed during the acute phase of myocardial infarction because plasma lipids undergo several phasic changes after myocardial infarction and hence may not reflect the patient's basal lipid state. $^{1-3}$ Consequently the standard recommendation is that lipids be assessed 2-3 months after the acute episode of myocardial infarction. ${ }^{3}$ This frequently delays or prevents any interventional programme and some patients may escape screening completely.

Recent studies seem to suggest that lipid state can be assessed accurately within 24 hours after

Correspondence: M. Sewdarsen M.B., Ch.B., Department of Medicine, R.K. Khan Hospital, Private Bag X004, Chatsworth, 4030 Durban, Natal, South Africa.

*Present address: Department of Medicine, Division of Metabolism, University of Washington, Seattle, Washington, USA.

Accepted: 22 December 1987 myocardial infarction and that contrary to common belief 3 months do not have to pass before evaluating plasma lipids. ${ }^{4-6}$ However, it is not clear from current literature whether this is true irrespective of glucose tolerance since lipid abnormalities are closely related to glucose tolerance. ${ }^{7}$ Furthermore the effect of the sex of the patient on these lipid levels has not been assessed. Hence the purpose of this study was to determine whether serum lipid values recorded soon after myocardial infarction reflected the patient's basal lipid state as recorded 3 months post infarction and whether this was true irrespective of gender and glucose tolerance.

\section{Patients}

Consecutive patients below the age of 61 years admitted with acute myocardial infarction within 24 hours of onset of symptoms were eligible for

(C) The Fellowship of Postgraduate Medicine, 1988 
inclusion in the study. The diagnosis of acute myocardial infarction was based on a typical history of prolonged chest pain, electrocardiographic changes and a diagnostic rise in creatinine kinase activity. Patients who died during the acute phase and those who did not return for follow-up were excluded from the final analyses. The study group hence consisted of 159 patients between the ages of 25 to 60 years (mean $48 \pm$ s.e.m. 0.64 years). There were 134 men (mean $48 \pm 0.71$ years) and 25 women (mean $51 \pm 1.42$ years). Quetelet's body mass index calculated by the formula weight $(\mathrm{kg}) /$ height $(\mathrm{m})^{2}$ was $24.29 \pm 0.32$ for men and $26.34 \pm 0.75$ for women $(P=0.01)$. On admission (day 1) a random, non-fasting specimen of blood was taken. The following morning (day 2) fasting samples were taken and repeated 3 months later. Concentrations of serum total cholesterol and triglycerides were measured in each sample by methods previously described. ${ }^{8} \mathrm{~A}$ modified oral glucose tolerance test was performed 3 months post-infarction in all patients not known to be diabetic. The results of the oral glucose tolerance tests were interpreted by WHO criteria. ${ }^{9}$ All patients were given standard advice on dietary measures and none were on lipid lowering agents. The significance of differences was assessed by using the paired $t$ test. All data are given as mean \pm s.e.m. In addition $95 \%$ confidence intervals (CI), calculated by the method of Gardner and Altman, ${ }^{10}$ are given for differences in means.

\section{Results}

Tables I and II give the results of the cholesterol analysis. The mean total cholesterol on admission was similar to the 3 month value in both sexes. The mean cholesterol fell significantly on day 2 in both sexes (males $P<0.0005,95 \% \mathrm{CI} 0.35$ to 0.57 ; females $P<0.0005,95 \%$ CI 0.23 to 0.66 ) and in men the day 2 value was significantly lower than the 3 month value $(P<0.0005,95 \%$ CI 0.41 to 0.69) whilst in women there was no significant difference. Glucose tolerance of the patients did not influence the cholesterol changes after myocardial infarction (Table II).

Not only was the admission mean cholesterol of the entire group similar to the values obtained at three months but the correlation coefficient, $r=0.66$, between these values was highly significant $(P<0.0005)$. Similar significant correlation coefficients were obtained between admission and 3 month cholesterol values in both men $(r=0.64$; $P<0.0005)$ and women $(r=0.73 ; P<0.0005)$ as well between day 2 and 3 month cholesterol results (men: $\quad r=0.70 ; \quad P<0.0005$; women: $r=0.67$; $P<0.0005)$.

Serum triglyceride concentrations for the entire group showed no significant change between day 2 and 3 months though differences were observed in the values for men (Table III). When the triglyceride values were examined according to the patients' glucose tolerance then patients with normal glucose tolerance had significantly higher triglyceride values at 3 months (Table IV) whilst there was no significant difference in patients with abnormal glucose tolerance (impaired glucose tolerance and diabetes).

Although in the total male population the mean triglyceride values were significantly higher at 3 months, further analyses revealed that this was also so only in men with normal glucose tolerance $(n=64$, mean $1.41 \pm 0.09$ vs $1.61 \mathrm{mmol} / 1 \pm 0.09 ; 95 \%$ CI 0.04 to $0.36 ; \bar{P}=0.01)$. In men with impaired glucose tolerance $(n=23$, mean $1.80 \pm 0.15$ vs $2.04 \pm 0.15 \mathrm{mmol} / \mathrm{l} ; 95 \%$ CI -0.06 to 0.55 ;) and diabetes $(n=47$, mean $1.95 \pm 0.15$ vs $2.15 \pm$ $0.15 \mathrm{mmol} / 1 ; 95 \%$ CI -0.06 to 0.44 ; ) the fasting day 2 triglycerides were similar to the 3 month values.

Table I Changes in serum total cholesterol in patients with myocardial infarction

\begin{tabular}{lcrr}
\hline & $\begin{array}{c}\text { Males } \\
(n=134)\end{array}$ & $\begin{array}{c}\text { Females } \\
(n=25)\end{array}$ & $\begin{array}{c}\text { Total } \\
(n=159)\end{array}$ \\
\hline Admission (day 1) & $6.09(0.10)$ & $6.75(0.30)$ & $6.20(0.10)$ \\
Day 2 (fasting) & $5.64(0.09)$ & $6.30(0.27)$ & $5.74(0.09)$ \\
At 3 months & $6.18(0.09)$ & $6.44(0.22)$ & $6.22(0.08)$ \\
$95 \%$ CI* & -0.07 to $0.25^{1}$ & -0.74 to $0.11^{1}$ & -0.12 to $0.17^{1}$ \\
$95 \%$ CI $\dagger$ & 0.41 to $0.69^{2}$ & -0.29 to $0.56^{1}$ & 0.35 to $0.62^{2}$ \\
\hline
\end{tabular}

Values are mean (s.e.m.) mmol/l.

*Confidence intervals of admission vs 3 month values.

†Confidence intervals of day 2 vs 3 month values.

${ }^{1} P=$ not significant; ${ }^{2} P<0.0005$. 
Table II Changes in serum mean total cholesterol in patients with myocardial infarction in relation to glucose tolerance

\begin{tabular}{lcrr}
\hline & $\begin{array}{c}\text { Normal } \\
(n=67)\end{array}$ & \multicolumn{1}{c}{$\begin{array}{c}\text { IGT } \\
(n=24)\end{array}$} & \multicolumn{1}{c}{$\begin{array}{c}\text { Diabetic } \\
(n=68)\end{array}$} \\
\hline Admission (day 1) & $6.18( \pm 0.14)$ & $6.18( \pm 0.23)$ & $6.22( \pm 0.16)$ \\
Day 2 & $5.75( \pm 0.14)$ & $5.92( \pm 0.20)$ & $5.67( \pm 0.14)$ \\
At 3 months & $6.22( \pm 0.13)$ & $6.40( \pm 0.17)$ & $6.17( \pm 0.13)$ \\
$95 \%$ CI* & -0.15 to $0.23^{1}$ & -0.17 to $0.61^{1}$ & -0.32 to $0.21^{1}$ \\
$95 \%$ CI $\dagger$ & 0.27 to $0.66^{3}$ & 0.11 to $0.85^{2}$ & 0.28 to $0.73^{4}$ \\
\hline
\end{tabular}

Values are mean (s.e.m.) mmol/l

*Admission vs 3 month values.

†Day 2 vs 3 month values.

IGT = impaired glucose tolerance; $\mathbf{C I}=$ confidence interval.

${ }^{1}$ Not significant; ${ }^{2} P<0.01 ;{ }^{3} P<0.001 ;{ }^{4} P<0.0005$.

Table III Changes in serum triglycerides in patients with myocardial infarction

\begin{tabular}{llllr}
\hline & Day 2 & 3 month & P value & $95 \%$ CI \\
\hline Males $(n=134)$ & $1.67(0.07)$ & $1.87(0.07)$ & $<0.001$ & 0.08 to 0.33 \\
Females $(n=25)$ & $2.65(0.30)$ & $2.27(0.17)$ & NS & -0.95 to 0.20 \\
Total $(n=159)$ & $1.82(0.08)$ & $1.94(0.07)$ & NS & -0.02 to 0.25 \\
\hline
\end{tabular}

All data are mean (s.e.m.) mmol/l.

$\mathrm{CI}=$ confidence intervals; $\mathrm{NS}=$ not significant.

Table IV Changes in serum triglyceride in patients with myocardial infarction in relation to glucose tolerance

\begin{tabular}{llcc}
\hline & $\begin{array}{c}\text { Normal } \\
\text { glucose } \\
\text { tolerance } \\
(n=67)\end{array}$ & $\begin{array}{c}I G T \\
(n=24)\end{array}$ & \multicolumn{1}{c}{$\begin{array}{c}\text { Diabetic } \\
(n=68)\end{array}$} \\
\hline Day 2 & $1.43(0.09)$ & $1.77(0.15)$ & $2.22(0.15)$ \\
At 3 months & $1.62(0.09)$ & $2.06(0.14)$ & $2.21(0.12)$ \\
$95 \%$ CI & 0.03 to $0.34^{2}$ & -0.01 to $0.59^{1}$ & -0.29 to $0.25^{1}$ \\
\hline
\end{tabular}

All data are mean (s.e.m.) mmol/l.

IGT = impaired glucose tolerance; $\mathbf{C I}=$ confidence interval.

${ }^{1} P=$ not significant; ${ }^{2} P<0.05$.

A significant correlation was demonstrated between day 2 and 3 month triglyceride results for both men $(r=0.65 ; \quad P<0.0005)$ and women $(r=0.43 ; P<0.05)$. Similar significant correlations were obtained irrespective of glucose tolerance (normal glucose tolerance: $n=67, \quad r=0.61$, $P=0.0001$; impaired glucose tolerance: $n=24$, $r=0.50, \quad P=0.01 ;$ diabetes: $n=68, \quad r=0.54$, $P=0.0003$.)

\section{Discussion}

In view of the accumulating evidence that treatment of hypercholesterolaemia may reduce mortality and morbidity after myocardial infarction ${ }^{11,12}$ it appears advantageous to screen and counsel hyperlipidaemic patients as soon as possible after an acute myocardial infarction at a time when patients and their families are most impressionable. Though recent literature suggests that lipids may be assessed within 24 hours after acute myocardial infarction ${ }^{4-6.13}$ and that 3 months need not pass before such assessment, the studies give no indication whether this is so irrespective of the gender and glucose tolerance of the patients.

The results of this study confirm and extend the 
findings of previous investigators that the total serum cholesterol ${ }^{4-6}$ concentrations measured within 24 hours after acute myocardial infarction are likely to reflect basal levels as determined 3 months post-infarction, and this relationship is independent of sex and glucose tolerance. Furthermore, in accord with other studies, we demonstrated a significant fall in serum cholesterol on day 2.4, However in contrast to the studies cited the day 2 cholesterol values in our study were significantly lower than the values obtained at 3 months. This was observed in men and was independent of glucose tolerance. In women cholesterol measured on day 2 did not differ from the 3 month values. The reasons for this are not clear but are probably related to differences in cholesterol metabolism. Obesity, which is commoner in Indian women than men with myocardial infarction, enhances cholesterol production. ${ }^{14}$ Also women tend to catabolise cholesterol less effectively than men. ${ }^{14}$ Both these mechanisms will tend to retard the expected decrease in cholesterol levels after infarction. Despite these changes in cholesterol on day 2, a significant correlation was demonstrated between day 2 and 3 month cholesterol values in both men and women.

With respect to triglycerides no significant differences were demonstrated between the fasting day 2 and the 3 month values for the whole group and this accords with other studies. ${ }^{4,5}$ However, we have demonstrated that triglyceride levels after myocardial infarction are influenced by glucose tolerance of the patients. In patients with normal glucose tolerance the day 2 levels were significantly lower than the 3 month levels whereas there was no difference in patients with abnormal glucose tolerance. Nevertheless a significant correlation was still demonstrable between the day 2 and 3 month values in patients with normal glucose tolerance. Comparison of triglyceride levels according to the sex of the patients showed a difference between day 2 and 3 month levels in men but not in women. This difference may be explained by the fact that

\section{References}

1. Friedrickson, D.S. The role of lipids in acute myocardial infarction. Circulation 1969, 39 \& 40: 99-111.

2. Dodds, C. \& Mills, G.L. Influence of myocardial infarction on plasma lipoprotein concentration. Lancet 1959, i: $1160-1163$.

3. Watson, W.C., Buchanon, K.D. \& Dickson, C. Serum cholesterol levels after myocardial infarction. $\mathrm{Br} \mathrm{Med}$ $J$ 1963, 2: 709-712. the majority of women (22/25) had abnormal glucose tolerance. Furthermore there was no difference between day 2 and 3 month triglyceride levels in men with abnormal glucose tolerance. Thus the behaviour of triglycerides after acute myocardial infarction appears to be influenced by the glucose tolerance and not the gender of the patients. The reasons for the difference in triglyceride levels between patients with normal and abnormal glucose tolerance are not clear and require further investigation. Whereas serum cholesterol concentrations fall soon after acute myocardial infarction, ${ }^{1,13}$ serum triglycerides tend to rise, and this occurs after a few days. ${ }^{1,15}$ Tibblin demonstrated that the peak rise occurred approximately after 3 weeks and declined to initial values after 12 months. ${ }^{15}$ It is possible that the elevated basal triglyceride levels associated with abnormal glucose tolerance mask the expected rise after acute myocardial infarction. Insulin activity is also affected after myocardial infarction and this may modify the behaviour of serum triglycerides after myocardial infarction. ${ }^{16}$

In conclusion this study has demonstrated that in patients with myocardial infarction, admission cholesterol levels may be taken to represent basal levels irrespective of the sex or glucose tolerance of the patients. The optimum time to measure cholesterol concentrations is thus immediately on admission. Although on the following morning the cholesterol in men and the triglycerides in patients with normal glucose tolerance are significantly lower than the 3 month values, the day 2 values are still a useful guide to the patients lipid state because of the significant correlation between cholesterol, triglycerides on day 2 and their respective 3 month values.

\section{Acknowledgements}

We thank the nursing staff in the coronary care unit and ischaemic heart disease clinic for their cooperation and $\mathrm{Dr}$ P.K. Naidoo, Medical Superintendent of R. K. Khan Hospital for permission to publish.

4. Fyfe, T., Baxter, R.H., Cochran, K.M. \& Booth, E.M. Plasma-lipid changes after myocardial infarction. Lancet 1971, i: 997-1001.

5. Ryder, R.E.J., Hayes, T.M., Mulligan, I.P., Kingswood, J.C., Williams, S. \& Owens, D.R. How soon after myocardial infarction should plasma lipid values be assessed? Br Med J 1984, 289: 1651-1653. 
6. Gore, J.M., Goldberg, R.J., Matsumoto, A.S., Castelli, W.P., McNamara, P.M. \& Dalen, J.E. Validity of serum total cholesterol level obtained within 24 hours of acute myocardial infarction. Am J Cardiol 1984, 54: 722-725.

7. Phillips, G.B. Relationship between serum sex hormones and glucose, insulin, and lipid abnormalities in men with myocardial infarction. Proc Natl Acad Sci USA 1977, 74: 1729-1733.

8. Sewdarsen, M., Jialal, I. \& Vythilingum, S. Abnormalities of glucose tolerance and lipid abnormalities in Indian myocardial infarct survivors. $S$ Afr Med J 1983, 63: 238-240.

9. World Health Organisation. Expert committee on diabetes mellitus. WHO Tech Rep Ser 1980; No. 646.

10. Gardner, M.J. \& Altman, D.G. Confidence intervals rather than $P$ values: estimation rather than hypothesis testing. $\mathrm{Br}$ Med J 1986, 292: 746-750.

11. Coronary Drug Project Research Group. Factors influencing long term prognosis in the coronary drug project. J Chronic Dis 1974, 27: 267-285.
12. Coronary Drug Project Research Group. Natural history of myocardial infarction in the Coronary Drug Project: long term prognostic importance of serum lipid levels. Am J Cardiol 1978, 42: 489-498.

13. Jackson, R., Scragg, R., Marshall, R., White, H., O'Brien, K. \& Small, C. Changes in serum lipid concentrations during first 24 hours after myocardial infarction. Br Med J 1987, 294: 1588-1589.

14. Miettinen, T.A. Current views on cholesterol metabolism. Horm Metab Res 1974, 6 (suppl 4): 37-44.

15. Tibblin, G. \& Cramer, K. Serum lipids during the course of an acute myocardial infarction and one year afterwards. Acta Med Scand 1963, 174: 451-455.

16. Ganda, O.P. Pathogenesis of macrovascular disease including the influence of lipids. In: Marble, A., Krall, L.P., Bradley, R.F. Christlieb, A.R. \& Soeldner, J.S. (eds) Joslin's Diabetes Mellitus, 12th ed. Lea \& Febiger, Philadelphia, 1985, pp. 217-250. 